\title{
Optimización del mecanizado de agujeros profundos de pequeño diámetro por electroerosión usando la metodología Taguchi
}

\author{
Small deep hole drilling electro discharge machining process \\ optimization using Taguchi method
}

Otimização da usinagem de buracos profundos de pequeno
diâmetro por eletroerosão usando a metodologia Taguchi

Fecha de recepción: 24 de septiembre de 2015

Guillermo Jiménez-Chavarro

Fecha de aprobación: 30 de marzo de 2016

Arthur José Vieira-Porto*

Roberto Hideaki-Tsunaki ${ }^{\text {*t+k }}$

\section{Resumen}

Realizar agujeros profundos de pequeño diámetro en metales de alta dureza es una operación que está fuera del alcance del proceso de perforación con arranque de viruta; uno de los procesos más adecuados para ello es el mecanizado por electroerosión. Debido a las múltiples variables involucradas en electroerosión y a las restricciones impuestas por las dimensiones reducidas, es necesario determinar el nivel de ajuste preciso de cada una de las variables para realizar un mecanizado eficiente y de buena calidad. Usando un arreglo ortogonal $\mathrm{L}_{27}$ Taguchi, fue posible analizar el efecto de las variables eléctricas, de limpieza y del diámetro del electrodo sobre las características del mecanizado de perforaciones de pequeño diámetro en piezas de acero rápido DIN 1.3344. El análisis de los resultados experimentales y su conversión a señales de ruido permitieron optimizar la tasa de remoción de material, la velocidad de avance de perforación, el desgaste del electrodo y la rugosidad superficial.

Palabras clave: acero rápido, electroerosión, método Taguchi, perforación profunda.

\footnotetext{
* M. Sc. Universidade de São Paulo (São Carlos, Brasil). guillermo.jimenez@usp.br.

** Ph. D. Universidade de São Paulo (São Carlos, Brasil). ajvporto@sc.usp.br.

*** Ph.D. Universidade de São Paulo (São Carlos, Brasil). rtsunaki@sc.usp.br.
} 


\section{Abstract}

Small deep hole drilling in high hardness metals is an operation beyond the reach of the conventional drilling machine process, and one of the most suitable processes for this operation is the Electro Discharge Machining, EDM. Due to the numerous variables involved in electro discharge machining and the restrictions imposed by the small size, it is necessary to determine the precise adjustment level for each of the variables in order to reach an efficient and good quality machining process.

This paper shows how using a Taguchi $\mathrm{L}_{27}$ orthogonal arrangement, the cleaning effect of the electrical variables, and the electrode diameter on the machining characteristics of small diameter holes in a DIN 1.3344 work piece, made it possible to be analyzed. The experimental analysis results and their data turned into noise signals, allowed to optimize the material removal rate, the feed rate drilling speed, the electrode wear and the surface roughness.

Keywords: Electro Discharge Machining (EDM), high speed steel, small and deep hole drilling, Taguchi method.

\section{Resumo}

Realizar buracos profundos de pequeno diâmetro em metais de alta dureza é uma operação que está fora do alcance do processo de perfuração com arranque de cavaco; um dos processos mais adequados para isso é a usinagem por eletroerosão. Devido às múltiplas variáveis envolvidas em eletroerosão e às restrições impostas pelas dimensões reduzidas, é necessário determinar o nível de ajuste preciso de cada uma das variáveis para realizar uma usinagem eficiente e de boa qualidade. Usando uma distribuição ortogonal L27 Taguchi, foi possível analisar o efeito das variáveis elétricas, de limpeza e do diâmetro do eletrodo sobre as características da usinagem de perfurações de pequeno diâmetro em peças de aço rápido DIN 1.3344. A análise dos resultados experimentais e sua conversão a sinais de ruído permitiram otimizar a taxa de remoção de material, a velocidade de avanço de perfuração, o desgaste do eletrodo e a rugosidade superficial.

Palavras chave: aço rápido, eletroerosão, método Taguchi, perfuração profunda.

Cómo citar este artículo:

[1] G. Jiménez-Chavarro, A. J. Vieira-Porto and R. Hideaki-Tsunaki, "Optimización del mecanizado de agujeros profundos de pequeño diámetro por electroerosión usando la metodología Taguchi”, Fac. Ing., vol. 25 (42), pp. 111-122, may.-ago. 2016. 


\section{INTRODUCCIÓN}

El mecanizado por electroerosión (EDM) es un proceso de naturaleza termoeléctrica mediante el cual el material de una pieza es removido mediante la aplicación de múltiples descargas eléctricas de corta duración por medio de un electrodo a través de un medio dieléctrico [1]. La realización de agujeros profundos en metales de alta dureza por electroerosión es una operación bastante utilizada en la industria aeroespacial, automotriz, médica y dental, en razón de su eficiencia y costo [2].

Las perforaciones profundas de pequeño diámetro son aquellas que tienen un diámetro entre 0,8 y $3 \mathrm{~mm}$ y una relación de aspecto mayor a 5 , y para su mecanizado por electroerosión, dadas las pequeñas dimensiones involucradas, es necesario realizar un ajuste preciso de las variables eléctricas y de limpieza del gap para que la operación de perforación tenga un rendimiento adecuado y buena calidad superficial [3].

Utilizando la metodología Taguchi, en este trabajo es analizado el efecto de las diferentes variables sobre las características de mecanizado de agujeros profundos de pequeño diámetro, con el objetivo de optimizar los resultados de ese proceso.

\section{MÉTODO EXPERIMENTAL}

\section{A. Materiales y equipos}

Las pruebas de perforación fueron realizadas utilizando una máquina $C N C$ de electroerosión por penetración
ELETRO ZNC-400; fueron utilizados electrodos tubulares de cobre electrolítico con diámetros entre 1,5 y $2,5 \mathrm{~mm}$, y como material de prueba se usaron barras de acero rápido DIN 1.3344 de sección cuadrada, de $12,7 \mathrm{~mm}$ de lado, cuyas propiedades están registradas en la Tabla 1. El líquido dieléctrico utilizado fue querosene, y para la limpieza del gap fue empleada inyección a presión $(9 \mathrm{psi})$ de dieléctrico por medio del conducto de cada electrodo. El montaje de las pruebas fue realizado como está ilustrado en la Fig. 1.

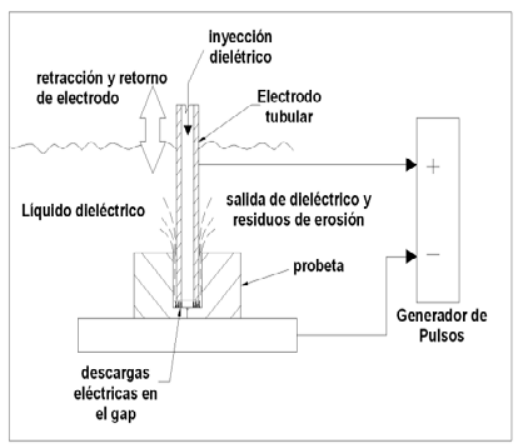

Fig. 1. Perforación profunda por EDM.

La rugosidad superficial fue medida utilizando un rugosímetro Taylor-Surf 5Q. El desgaste de los electrodos $(T W R)$ y la tasa de remoción de material $(M R R)$ fueron calculadas mediante las ecuaciones $1 \mathrm{y}$ 2.

$$
\begin{gathered}
T W R=\frac{\Delta L \text { electrodo }}{\text { profundidad de perforación }}(1) \\
M R R=\frac{\text { Área agujero } * \text { profundidad }}{\text { tiempo de perforación }}
\end{gathered}
$$

\begin{tabular}{|l|}
\multicolumn{1}{c}{ TABLA 1 } \\
\multicolumn{1}{c|}{ PropIEDADES HSS DIN 1.3344 } \\
\begin{tabular}{|l|l|}
\hline Composición & $1.17 \% \mathrm{C}, 0.30 \% \mathrm{Mn}, 4.25 \% \mathrm{Cr}, 5 \% \mathrm{Mo}, 6 \% \mathrm{~W}, 2.8 \% \mathrm{~V}$ \\
\hline Dureza & $66 \mathrm{HRC}$ \\
\hline
\end{tabular}
\end{tabular}

\section{B. Diseño del experimento}

El experimento fue diseñado con base en la metodología Taguchi. Se seleccionó un arreglo ortogonal L27 para analizar el efecto de siete variables en tres niveles, cada una sobre las características del proceso de perforación por EDM. Las variables y sus niveles y el arreglo experimental están registrados en las Tablas 2 y 3 , respectivamente. 


\section{TABLA 2}

ARREGLO ORTOGONAL L27 PARA 7 VARIABLES

\begin{tabular}{|c|c|c|c|c|c|c|c|}
\hline Prueba & A & B & C & D & $\mathbf{E}$ & $\mathbf{F}$ & $\mathbf{G}$ \\
\hline 1 & 1 & 1 & 1 & 1 & 1 & 1 & 1 \\
\hline 2 & 1 & 1 & 1 & 1 & 2 & 2 & 2 \\
\hline 3 & 1 & 1 & 1 & 1 & 3 & 3 & 3 \\
\hline 4 & 1 & 2 & 2 & 2 & 1 & 1 & 1 \\
\hline 5 & 1 & 2 & 2 & 2 & 2 & 2 & 2 \\
\hline 6 & 1 & 2 & 2 & 2 & 3 & 3 & 3 \\
\hline 7 & 1 & 3 & 3 & 3 & 1 & 1 & 1 \\
\hline 8 & 1 & 3 & 3 & 3 & 2 & 2 & 2 \\
\hline 9 & 1 & 3 & 3 & 3 & 3 & 3 & 3 \\
\hline 10 & 2 & 1 & 2 & 3 & 1 & 2 & 3 \\
\hline 11 & 2 & 1 & 2 & 3 & 2 & 3 & 1 \\
\hline 12 & 2 & 1 & 2 & 3 & 3 & 1 & 2 \\
\hline 13 & 2 & 2 & 3 & 1 & 1 & 2 & 3 \\
\hline 14 & 2 & 2 & 3 & 1 & 2 & 3 & 1 \\
\hline 15 & 2 & 2 & 3 & 1 & 3 & 1 & 2 \\
\hline 16 & 2 & 3 & 1 & 2 & 1 & 2 & 3 \\
\hline 17 & 2 & 3 & 1 & 2 & 2 & 3 & 1 \\
\hline 18 & 2 & 3 & 1 & 2 & 3 & 1 & 2 \\
\hline 19 & 3 & 1 & 3 & 2 & 1 & 3 & 2 \\
\hline 20 & 3 & 1 & 3 & 2 & 2 & 1 & 3 \\
\hline 21 & 3 & 1 & 3 & 2 & 3 & 2 & 1 \\
\hline 22 & 3 & 2 & 1 & 3 & 1 & 3 & 2 \\
\hline 23 & 3 & 2 & 1 & 3 & 2 & 1 & 3 \\
\hline 24 & 3 & 2 & 1 & 3 & 3 & 2 & 1 \\
\hline 25 & 3 & 3 & 2 & 1 & 1 & 3 & 2 \\
\hline 26 & 3 & 3 & 2 & 1 & 2 & 1 & 3 \\
\hline 27 & 3 & 3 & 2 & 1 & 3 & 2 & 1 \\
\hline
\end{tabular}




\section{TABLA 3}

NIVELES PARA LAS VARIABLES EXPERIMENTO L27

\begin{tabular}{llccc}
\hline & Variable & \multicolumn{3}{c}{ Nivel } \\
\cline { 3 - 5 } & & $\mathbf{1}$ & $\mathbf{2}$ & $\mathbf{3}$ \\
\hline $\mathbf{A}$ & Diámetro $(\mathrm{mm})$ & 1,5 & 2 & 2,5 \\
$\mathbf{B}$ & $\mathrm{I}_{\mathrm{e}}(\mathrm{A})^{1}$ & 3,33 & 5 & 6,66 \\
$\mathbf{C}$ & $\mathrm{T}_{\text {on }}(\mu \mathrm{s})^{2}$ & 25 & 50 & 75 \\
$\mathbf{D}$ & $\mathrm{T}_{\text {off }}(\mu \mathrm{s})^{3}$ & 15 & 30 & 45 \\
$\mathbf{E}$ & $\mathrm{D}_{\text {aff }}(\mathrm{mm})^{4}$ & 4 & 5 & 6 \\
$\mathbf{F}$ & $\mathrm{T}_{\text {er }}(\mathrm{s})^{5}$ & 1,2 & 1,5 & 1,8 \\
$\mathbf{G}$ & $\mathrm{V}_{\text {gap }}(\mathrm{V})^{6}$ & 30 & 40 & 50 \\
\hline
\end{tabular}

\section{Procedimiento de análisis de los resultados}

Los resultados de las pruebas fueron analizados utilizando la metodología Taguchi, por medio de la transformación de los datos en factores de señal de ruido $(S / N)$. El factor $S / N$ permite medir los efectos de una variable sobre la media y sobre la variabilidad de un proceso de manera simultánea [4].

Para la optimización de las características estudiadas, fueron utilizadas las transformaciones en $S / N$, mayor es mejor y menor es mejor para maximización y minimización de las respuestas, respectivamente. El cálculo de los factores $S / N$ se realizó mediante las siguientes ecuaciones 3 y 4 :

Menor es mejor:

$$
S / N=-10 \log _{10} \sum \frac{Y_{i}^{2}}{n}
$$

Mayor es mejor:

$$
S / N=-10 \log _{10}\left[\frac{1}{n} \sum \frac{1}{Y_{i}}\right]
$$

\section{RESULTAdOS Y DISCUSIÓN}

Las características estudiadas en este trabajo fueron la $M R R$, Avance de perforación, $T W R$, profundidad de las perforaciones, gap diametral y conicidad. Los resultados de cada prueba y sus transformaciones en $S / N$ están registrados en la Tabla 4.

\begin{tabular}{|c|c|c|c|c|c|c|c|c|c|c|c|}
\hline \multirow{2}{*}{$\begin{array}{l}\text { Prueba } \\
1\end{array}$} & \multicolumn{2}{|c|}{$\begin{array}{c}M R R(\mathbf{m m} 3 / \\
\text { min) }\end{array}$} & \multirow{2}{*}{$\begin{array}{c}S / N \\
-11,46\end{array}$} & \multicolumn{2}{|c|}{$\begin{array}{l}\text { Avance (mm/ } \\
\text { min) }\end{array}$} & \multirow{2}{*}{$\frac{S / N}{-16,41}$} & \multicolumn{2}{|c|}{$\begin{array}{c}\text { Profundidad } \\
(\mathrm{mm})\end{array}$} & \multirow{2}{*}{$\frac{S / \boldsymbol{N}}{22,08}$} & TWR (\%) & \multirow{2}{*}{$\frac{S / N}{10,950}$} \\
\hline & 0,267 & 0,267 & & 0,151 & 0,151 & & 12,70 & 12,70 & & $28,3 \% \quad 28,3 \%$ & \\
\hline 2 & 0,335 & 0,335 & $-9,50$ & 0,190 & 0,190 & $-14,45$ & 12,70 & 12,70 & 22,08 & $\%$ & 10,255 \\
\hline 3 & 0,307 & 0,307 & $-10,24$ & 0,174 & 0,174 & -15 & 12,70 & 12,70 & 22,08 & $22,8 \%$ & 12,828 \\
\hline 4 & 0,343 & 0,343 & $-9,30$ & 0,194 & 0,194 & $-14,24$ & 8,15 & 8,15 & 18,22 & $31,9 \%$ & 9,924 \\
\hline 5 & 0,701 & 0,701 & $-3,08$ & 0,397 & 0,397 & $-8,03$ & 12,70 & 12,70 & 22,08 & $23,6 \%$ & 12,534 \\
\hline 6 & 0,458 & 0,499 & $-6,43$ & 0,259 & 0,282 & $-11,37$ & 12,70 & 12,70 & 22,08 & $31,1 \% \quad 26,0 \%$ & 10,855 \\
\hline
\end{tabular}

\section{TABLA 4}

\footnotetext{
Intensidad máxima del pulso de energía.

Duración del pulso de energía.

Intervalo entre dos pulsos de energía.

Distancia de retracción periódica del electrodo.

Tiempo de erosión de material.

Voltaje en el gap de descarga.
} 


\begin{tabular}{|c|c|c|c|c|c|c|c|c|c|c|c|c|}
\hline \multirow{2}{*}{$\frac{\text { Prueba }}{7}$} & \multicolumn{2}{|c|}{$\begin{array}{c}M R R(\mathrm{~mm} 3 / \\
\min )\end{array}$} & \multirow{2}{*}{$\begin{array}{c}\boldsymbol{S} / \boldsymbol{N} \\
-2,23\end{array}$} & \multicolumn{2}{|c|}{$\begin{array}{l}\text { Avance (mm/ } \\
\text { min) }\end{array}$} & \multirow{2}{*}{$\frac{S / N}{-7,02}$} & \multicolumn{2}{|c|}{$\begin{array}{c}\text { Profundidad } \\
(\mathbf{m m})\end{array}$} & \multirow{2}{*}{$\frac{S / N}{22,08}$} & \multicolumn{2}{|c|}{ TWR (\%) } & \multirow{2}{*}{$\frac{\boldsymbol{S} / \boldsymbol{N}}{8,345}$} \\
\hline & 0,774 & 0,774 & & 0,454 & 0,438 & & 12,70 & 12,70 & & $40,6 \%$ & $35,8 \%$ & \\
\hline 8 & 0,701 & 0,701 & $-3,08$ & & & $-8,03$ & 12,70 & & 22,08 & $32,7 \%$ & & 9,715 \\
\hline 9 & 641 & 0,641 & & & & & 12,70 & & & $3,5 \%$ & & \\
\hline 10 & 0,469 & 0,469 & $-6,57$ & 8 & 108 & 36 & 12,70 & 12,70 & & $28,0 \%$ & & 11,072 \\
\hline 11 & 0,493 & 0,493 & $-6,15$ & 57 & 57 & & 12,70 & 12,70 & & $20,5 \%$ & & 3,777 \\
\hline 12 & 0,324 & 0,268 & $-10,68$ & 0,103 & 0,085 & $-20,62$ & 12,70 & 10,25 & 21,05 & $16,1 \%$ & $18,0 \%$ & 15,329 \\
\hline 13 & 1,247 & 1,247 & & & & & 12,70 & 12 & & & & \\
\hline 14 & 0,928 & 0,928 & -06 & 0,2 & 0,295 & & 12,70 & 12,70 & & & & \\
\hline 15 & 0,509 & 0,509 & -5 , & 0,1 & & & 10,05 & 10 & & & & \\
\hline 16 & 1,050 & 0,997 & 0,19 & 0,334 & 0,318 & $-9,7$ & 12,70 & 12,70 & 22,08 & $36,6 \%$ & 39,4 & 8,400 \\
\hline 17 & 1,023 & 1,023 & & 0, & & -9 & 12,70 & 12,70 & & & & 3,184 \\
\hline 18 & 0,767 & 0,767 & $-2,31$ & 0,226 & 0,226 & & 12,45 & 12,45 & & $39,4 \%$ & & \\
\hline 19 & 0,365 & 0,365 & $-8,76$ & 0,0 & 0,074 & -22 & 5,65 & & & $13,3 \%$ & & 17,540 \\
\hline 20 & 0,291 & 0,291 & & & & & 3,50 & U, & & $10,0 \%$ & & \\
\hline 21 & 0,266 & 0,326 & $-10,70$ & 0,054 & 0,066 & $-24,52$ & 3,80 & 7,30 & 56 & $5,3 \%$ & $8,2 \%$ & 23,221 \\
\hline 22 & 0,423 & 0,423 & $-7,48$ & 0,086 & 0,086 & $-21,30$ & 12,40 & 12,40 & 21,87 & $23,4 \%$ & $23,4 \%$ & 12,620 \\
\hline 23 & 0,561 & & & & & & 11,20 & & & & & \\
\hline 24 & 0,863 & 0,863 & $-1,28$ & & & & 10,90 & 10,90 & 20,75 & $33,0 \%$ & & 9,622 \\
\hline 25 & 1,094 & 1,094 & 0,78 & 0,223 & 0,223 & $-13,04$ & 12,70 & 12,70 & 22,08 & $24,4 \%$ & $24,4 \%$ & 12,249 \\
\hline 26 & 1,189 & 1,222 & 1,62 & 0,242 & 0,249 & $-12,20$ & 9,45 & 12,70 & 20,61 & $20,6 \%$ & $22,8 \%$ & 13,246 \\
\hline 27 & 1,383 & 1,383 & 2,82 & 0,282 & 0,282 & $-11,00$ & 12,40 & 12,40 & 21,87 & $26,6 \%$ & $26,6 \%$ & 11,498 \\
\hline
\end{tabular}

\section{A. MRR}

Los valores de $S / N$ registrados en la Fig. 2 mostraron que las variables con mayor efecto sobre la $M R R$ fueron $\mathrm{I}_{\mathrm{e}}$, diámetro de electrodo $\mathrm{y}_{\mathrm{er}}$.

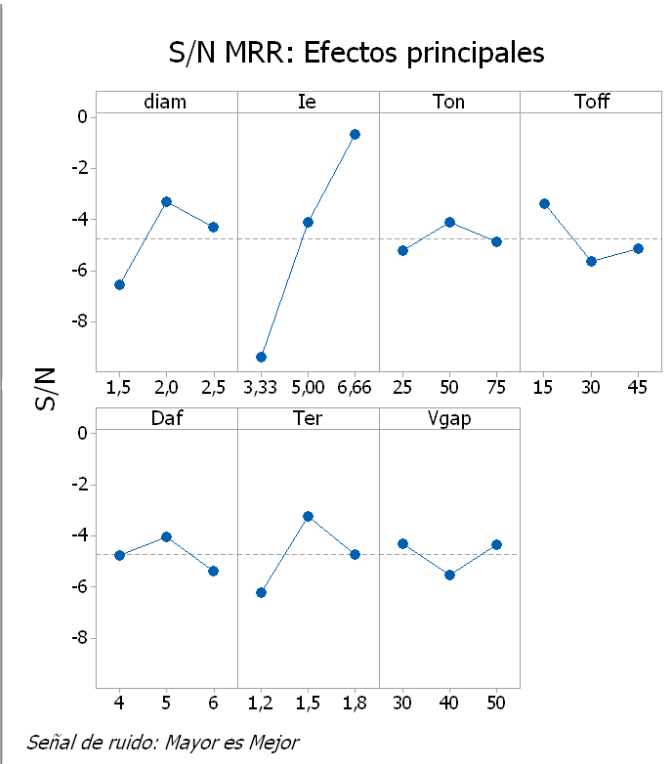

Fig. 2. S/N MRR.
El grado de relevancia de cada variable $(\Delta)$ y el valor de $S / N$ para cada uno de sus niveles están registrados en la Tabla 5.

\section{TABLA 5}

MRR: SEÑALES DE RUIDO

\begin{tabular}{|c|c|c|c|c|c|}
\hline \multicolumn{6}{|c|}{$S / N M R R$} \\
\hline \multirow{2}{*}{ Variable } & \multicolumn{3}{|c|}{ Nível } & \multirow{2}{*}{$\Delta$} & \multirow{2}{*}{ Rank } \\
\hline & 1 & 2 & 3 & & \\
\hline $\mathrm{I}_{\mathrm{e}}$ & $-9,42$ & $-4,131$ & $-0,651$ & 8,769 & 1 \\
\hline Diámetro & $-6,576$ & $-3,323$ & $-4,304$ & 3,253 & 2 \\
\hline $\mathrm{T}_{\text {er }}$ & $-6,216$ & $-3,254$ & $-4,733$ & 2,962 & 3 \\
\hline $\mathrm{T}_{\text {off }}$ & $-3,398$ & $-5,655$ & $-5,15$ & 2,257 & 4 \\
\hline $\mathrm{D}_{\mathrm{af}}$ & $-4,767$ & $-4,042$ & $-5,393$ & 1,351 & 5 \\
\hline $\mathrm{V}_{\text {gap }}$ & $-4,306$ & $-5,552$ & $-4,345$ & 1,246 & 6 \\
\hline $\mathrm{T}_{\text {on }}$ & $-5,211$ & $-4,11$ & $-4,882$ & 1,101 & 7 \\
\hline
\end{tabular}

Los efectos de cada variable analizada sobre la $M R R$ fueron coherentes con la información en la literatura $[5,6]$. En la Fig. 2 se observa que el incremento de $\mathrm{I}_{\mathrm{e}} \mathrm{y}$ la reducción de duración de $\mathrm{T}_{\text {on }}$ produjeron incremento de la $M R R$ media, y que la utilización del menor diámetro de electrodos causó una reducción significativa de la MRR. Los resultados también 
indican que $\mathrm{T}_{\text {er }}$ debe tener un valor intermedio para maximizar la remoción de material, de modo que la cantidad de residuos producidos no desestabilice el proceso de mecanizado, pero sí que tenga la mayor duración posible entre retracciones.
Los resultados indicaron que la variación de $\mathrm{T}_{\text {on }}$ no causó un efecto apreciable en la media de la MRR. Ese efecto pudo ser causado por los cambios que ejerce el diámetro de los electrodos utilizados sobre la duración de $\mathrm{T}_{\text {on }}$, para la cual se presenta $M R R$ máxima, la cual disminuye con la reducción del diámetro del electrodo $[7,8]$.

\section{TABLA 6}

MRR VALORES MEDIOS

\begin{tabular}{|c|c|c|c|c|c|}
\hline \multirow{3}{*}{ VARIABLE } & \multicolumn{5}{|c|}{ MÉDIA MRR $\left(\mathrm{mm}^{3} / \mathrm{min}\right)$} \\
\hline & \multicolumn{3}{|c|}{ Nivel } & \multirow{2}{*}{$\Delta$} & \multirow{2}{*}{ Rank } \\
\hline & 1 & 2 & 3 & & \\
\hline Diámetro & 0,5054 & 0,7507 & 0,7202 & 0,2453 & 2 \\
\hline $\mathrm{I}_{\mathrm{e}}$ & 0,3467 & 0,6726 & 0,957 & 0,6103 & 1 \\
\hline $\mathrm{T}_{\text {on }}$ & 0,6189 & 0,7182 & 0,6392 & 0,0993 & 6 \\
\hline $\mathrm{T}_{\text {off }}$ & 0,8085 & 0,5876 & 0,5802 & 0,2283 & 3 \\
\hline $\mathrm{D}_{\mathrm{af}}$ & 0,6672 & 0,6932 & 0,6158 & 0,0774 & 7 \\
\hline $\mathrm{T}_{\mathrm{er}}$ & 0,5572 & 0,78 & 0,6391 & 0,2228 & 4 \\
\hline $\mathrm{V}_{\mathrm{gar}}$ & 0,7078 & 0,5768 & 0,6917 & 0,131 & 5 \\
\hline
\end{tabular}

El valor máximo de $M R R\left(1.383 \mathrm{~mm}^{3} / \mathrm{min}\right)$ fue logrado bajo las condiciones del test 27. Mediante el análisis de los valores de $S / N$ de cada variable fue posible predecir que podría ser obtenida una $M R R$ máxima mediante la combinación de variables $\mathrm{A}_{2} \mathrm{~B}_{3} \mathrm{C}_{2} \mathrm{D}_{1} \mathrm{E}_{2} \mathrm{~F}_{2} \mathrm{G}_{2}$. El resultado obtenido con la predicción y el grado de optimización están registrados en la Tabla 9.

\section{B. Velocidad de avance de perforación}

El análisis de los valores de $S / N$ indicó que las variables con mayor efecto sobre la velocidad de avance fueron $\mathrm{I}_{\mathrm{e}}$, diámetro de electrodo y $\mathrm{T}_{\mathrm{er}}$. El grado de relevancia de cada variable $(\Delta)$ y el valor de $S / N$ para cada uno de sus niveles están registrados en la Tabla 7 y en la Fig. 3.

\section{TABLA 7}

Avance: SEÑALES DE RUIDO

\begin{tabular}{lccccc}
\hline \multicolumn{5}{c}{ AVANCE $(\boldsymbol{S} / \mathbf{N})$} \\
Nível & & & \\
\cline { 2 - 4 } VARIABLE & $\mathbf{1}$ & $\mathbf{2}$ & $\mathbf{3}$ & $\boldsymbol{\Delta}$ & Rank \\
\cline { 2 - 4 } $\mathrm{I}_{\mathrm{e}}$ & $-19,306$ & $-13,775$ & $-10,277$ & 9,029 & 1 \\
Diámetro & $-11,504$ & $-13,656$ & $-18,198$ & 6,694 & 2 \\
$\mathrm{~T}_{\text {er }}$ & $-15,842$ & $-13,214$ & $-14,302$ & 2,628 & 3 \\
$\mathrm{~T}_{\text {off }}$ & $-12,968$ & $-15,297$ & $-15,094$ & 2,329 & 4 \\
$\mathrm{D}_{\text {af }}$ & $-14,636$ & $-13,611$ & $-15,111$ & 1,5 & 5 \\
$\mathrm{~V}_{\text {gap }}$ & $-13,933$ & $-15,194$ & $-14,231$ & 1,261 & 6 \\
$\mathrm{~T}_{\text {on }}$ & $-14,928$ & $-13,996$ & $-14,435$ & 0,932 & 7 \\
\hline
\end{tabular}




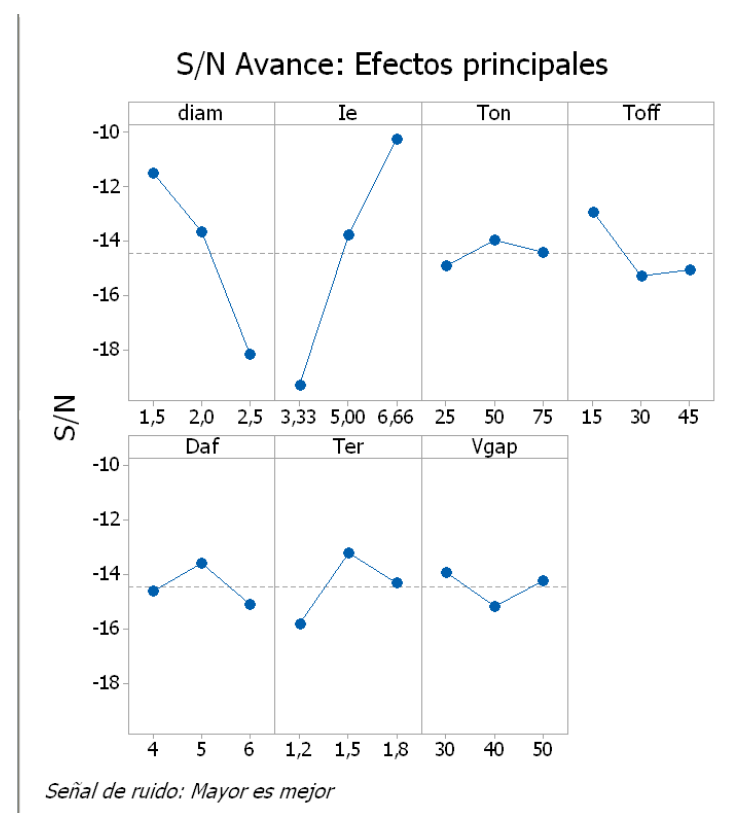

Fig. 3. S/N Avance.

La velocidad de avance de perforación tuvo un comportamiento similar al de la $M R R$, ya que son mediciones estrechamente relacionadas. La diferencia observada entre las dos características consistió en que la reducción del diámetro provocó incremento en la velocidad de avance promedio, como está registrado en la Tabla 8. El orden de grado de relevancia de las variables para el avance fue el mismo obtenido para la $M R R$.

\section{TABLA 8}

AVANCE: VALORES MEDIOS

\begin{tabular}{|c|c|c|c|c|c|}
\hline \multirow{3}{*}{ VARIABLE } & \multicolumn{5}{|c|}{ Media Avance (mm/min) } \\
\hline & \multicolumn{3}{|c|}{ Nivel } & \multirow{2}{*}{$\Delta$} & \multirow{2}{*}{ Rank } \\
\hline & 1 & 2 & 3 & & \\
\hline Diámetro & 0,2869 & 0,2323 & 0,1453 & 0,1416 & 2 \\
\hline $\mathrm{I}_{\mathrm{e}}$ & 0,1186 & 0,231 & 0,3148 & 0,1962 & 1 \\
\hline $\mathrm{T}_{\text {on }}$ & 0,1951 & 0,219 & 0,2504 & 0,0553 & 4 \\
\hline $\mathrm{T}_{\text {off }}$ & 0,2355 & 0,2148 & 0,2141 & 0,0214 & 7 \\
\hline $\mathrm{D}_{\mathrm{af}}$ & 0,2227 & 0,2423 & 0,1995 & 0,0428 & 5 \\
\hline $\mathrm{T}_{\text {er }}$ & 0,1881 & 0,2576 & 0,2187 & 0,0695 & 3 \\
\hline $\mathrm{V}_{\text {gap }}$ & 0,2304 & 0,2055 & 0,2286 & 0,0249 & 6 \\
\hline
\end{tabular}

La máxima velocidad de avance de perforación $(0.45 \mathrm{~mm} / \mathrm{min})$ fue lograda bajo las condiciones del test 7. Mediante el análisis de los valores de $S / N$ fue predicho que podría maximizarse la velocidad de avance utilizando la combinación de variables $\mathrm{A}_{1} \mathrm{~B}_{3} \mathrm{C}_{2} \mathrm{D}_{2} \mathrm{E}_{1} \mathrm{~F}_{1} \mathrm{G}_{1}$. El resultado obtenido con la combinación predicha y el grado de optimización están registrados en la Tabla 9. 


\section{TABLA 9}

OPTIMIZACIÓN DE CARACTERÍSTICAS DE LAS PERFORACIONES

\begin{tabular}{|c|c|c|c|c|c|c|c|c|c|c|}
\hline Característica & Prueba & Diam & $\mathbf{I}_{\mathrm{e}}$ & $\mathbf{T}_{\text {on }}$ & $\mathbf{T}_{\text {off }}$ & $\mathbf{D}_{\mathrm{af}}$ & $\mathbf{T}_{\text {er }}$ & $\mathbf{V}_{\text {gap }}$ & Valor & Mejora \\
\hline \multirow{2}{*}{$\operatorname{MRR}\left(\mathrm{mm}^{3} / \mathrm{min}\right)$} & 27 & 2,5 & 2 & 50 & 15 & 6 & 1,5 & 30 & 1,383 & \multirow{2}{*}{$25 \%$} \\
\hline & Predicción & 2 & 2 & 50 & 15 & 5 & 1,5 & 30 & 1,735 & \\
\hline \multirow{2}{*}{ Avance $(\mathrm{mm} / \mathrm{min})$} & 7 & 1,5 & 2 & 75 & 45 & 4 & 1,2 & 30 & 0,45 & \multirow{2}{*}{$33 \%$} \\
\hline & Predicción & 1,5 & 2 & 50 & 15 & 5 & 1,5 & 30 & 0,6 & \\
\hline \multirow{2}{*}{$T W R(\%)$} & 21 & 2,5 & 1 & 75 & 30 & 6 & 1,5 & 30 & $5,3 \%$ & \multirow{2}{*}{$3 \%$} \\
\hline & Predicción & 2,5 & 1 & 75 & 30 & 6 & 1,5 & 40 & $5,1 \%$ & \\
\hline \multirow{2}{*}{ 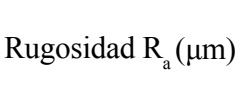 } & 22 & 2,5 & 1,5 & 25 & 45 & 4 & 1,8 & 40 & 2,82 & \multirow{2}{*}{$6 \%$} \\
\hline & Predicción & 2,5 & 1 & 25 & 45 & 6 & 1,5 & 40 & 2,65 & \\
\hline
\end{tabular}

\section{TWR}

El análisis de los valores de $S / N$ indicó que las variables con mayor efecto sobre la $T W R$ fueron Ie,
$\mathrm{T}_{\text {on }}$ y diámetro de electrodo. El grado de relevancia de cada variable $(\Delta)$ y el valor de $S / N$ para cada uno de sus niveles están registrados en la Tabla 10.

TABla 10

TWR: SEÑALES DE RUIDO

\section{$S / N$ DESGASTE}

\begin{tabular}{|c|c|c|c|c|c|}
\hline \multirow{2}{*}{ VARIABLE } & \multicolumn{3}{|c|}{ Nivel } & \multirow{2}{*}{$\Delta$} & \multirow{2}{*}{ Rank } \\
\hline & 1 & 2 & 3 & & \\
\hline$I_{e}$ & 14,996 & 11,739 & 9,916 & 5,08 & 1 \\
\hline $\mathrm{T}_{\text {on }}$ & 10,001 & 12,275 & 14,375 & 4,374 & 2 \\
\hline Diámetro & 10,546 & 11,767 & 14,338 & 3,792 & 3 \\
\hline $\mathrm{T}_{\text {off }}$ & 12,452 & 13,195 & 11,005 & 2,19 & 4 \\
\hline $\mathrm{D}_{\mathrm{af}}$ & 11,814 & 12,038 & 12,801 & 0,987 & 5 \\
\hline $\mathrm{V}_{\text {gap }}$ & 11,9 & 12,509 & 12,243 & 0,609 & 6 \\
\hline $\mathrm{T}_{\mathrm{er}}$ & 12,132 & 12,393 & 12,126 & 0,267 & 7 \\
\hline
\end{tabular}

Los efectos producidos por $\mathrm{I}_{\mathrm{e}} \mathrm{y}_{\mathrm{on}}$ correspondieron a lo indicado en la literatura y en diferentes trabajos de investigación [6,9]. En la Fig. 4 puede ser observado que el incremento de $\mathrm{I}_{\mathrm{e}}$ y la reducción de $\mathrm{T}_{\text {on }} \mathrm{y}$ del diámetro del electrodo produjeron un incremento significativo del desgaste de los electrodos.

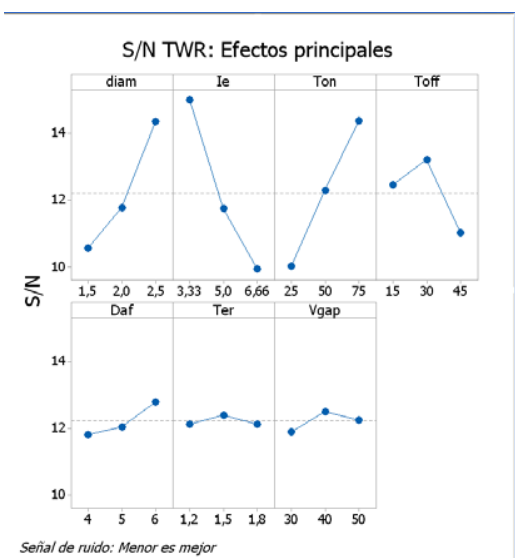

Fig. 4. S/N desgaste. 
Los resultados del experimento mostraron que la reducción de $\mathrm{T}_{\text {off }} \mathrm{y}$ el incremento de la distancia de retracción del electrodo tuvieron efecto en la reducción de la $T W R$. Las variables de limpieza, al igual que $\mathrm{V}_{\text {gap }}$, presentaron un efecto imperceptible sobre el desgaste de los electrodos, como está registrado en la Tabla 11.

\section{TABLA 11}

TWR: VALORES MEDIOS

\begin{tabular}{lccccc}
\hline \multirow{2}{*}{ Variable } & \multicolumn{5}{c}{ MEDIA Desgaste (\%) } \\
\cline { 2 - 4 } & $\mathbf{1}$ & $\mathbf{2}$ & $\mathbf{3}$ & $\boldsymbol{\Delta}$ & Rank \\
\hline Diámetro & $30,03 \%$ & $27,22 \%$ & $21,61 \%$ & $8,42 \%$ & 3 \\
$\mathrm{I}_{\mathrm{e}}$ & $19,71 \%$ & $26,54 \%$ & $32,60 \%$ & $12,89 \%$ & 1 \\
$\mathrm{~T}_{\text {on }}$ & $32,21 \%$ & $24,70 \%$ & $21,94 \%$ & $10,27 \%$ & 2 \\
$\mathrm{~T}_{\text {off }}$ & $24,19 \%$ & $25,60 \%$ & $29,06 \%$ & $4,87 \%$ & 4 \\
$\mathrm{D}_{\text {af }}$ & $26,98 \%$ & $26,65 \%$ & $25,23 \%$ & $1,75 \%$ & 6 \\
$\mathrm{~T}_{\text {er }}$ & $26,81 \%$ & $26,30 \%$ & $25,75 \%$ & $1,06 \%$ & 7 \\
$\mathrm{~V}_{\text {gap }}$ & $27,85 \%$ & $24,88 \%$ & $26,12 \%$ & $2,97 \%$ & 5 \\
\hline
\end{tabular}

El valor mínimo de TWR obtenido en el experimento $(5,3 \%)$ fue alcanzado sobre las condiciones del test 21. Mediante el análisis de $S / N$ fue predicho que podría obtenerse un valor de $T W R$ mínimo en una perforación utilizando la combinación de variables $\mathrm{A}_{1} \mathrm{~B}_{3} \mathrm{C}_{2} \mathrm{D}_{2} \mathrm{E}_{1} \mathrm{~F}_{1} \mathrm{G}_{2}$. El resultado logrado utilizando la combinación predicha y el grado de optimización están registrados en la Tabla 9.

\section{Rugosidad superficial}

El análisis de los valores de $S / N$ indicó que las variables con mayor efecto sobre la Rugosidad superficial fueron Ie, $\mathrm{T}_{\text {on }}$ y $\mathrm{D}_{\text {af }}$ El grado de relevancia de cada variable $(\Delta)$ y el valor de $S / N$ para cada uno de sus niveles están registrados en la Tabla 12 y en la Fig. 5.

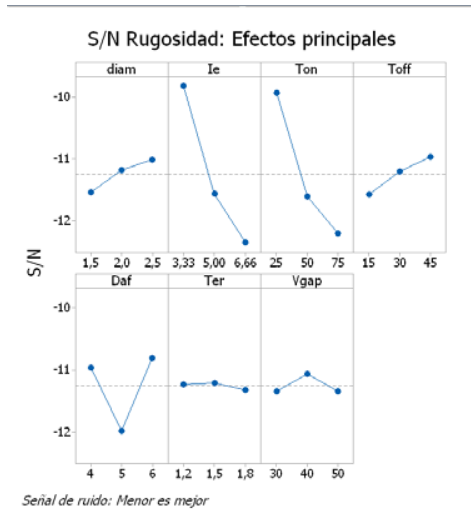

Fig. 5. S/N Rugosidad superficial.

\section{TABLA 12}

RUGOSIDAD: SEÑALES DE RUIDO

S/N RUGOSIDADSUPERFICIAL

\begin{tabular}{|c|c|c|c|c|c|}
\hline \multirow{2}{*}{ VARIABLE } & \multicolumn{3}{|c|}{ Nivel } & \multirow{2}{*}{$\Delta$} & \multirow{2}{*}{ Rank } \\
\hline & 1 & 2 & 3 & & \\
\hline$I_{e}$ & $-9,831$ & $-11,56$ & $-12,355$ & 2,524 & 1 \\
\hline $\mathrm{T}_{\text {on }}$ & $-9,937$ & $-11,606$ & $-12,203$ & 2,266 & 2 \\
\hline $\mathrm{D}_{\mathrm{af}}$ & $-10,964$ & $-11,98$ & $-10,802$ & 1,178 & 3 \\
\hline $\mathrm{T}_{\text {off }}$ & $-11,557$ & $-11,204$ & $-10,965$ & 0,592 & 4 \\
\hline Diámetro & $-11,547$ & $-11,179$ & $-11,02$ & 0,527 & 5 \\
\hline$V_{\text {gap }}$ & $-11,341$ & $-11,061$ & $-11,344$ & 0,283 & 6 \\
\hline $\mathrm{T}_{\mathrm{er}}$ & $-11,228$ & $-11,201$ & $-11,318$ & 0,117 & 7 \\
\hline
\end{tabular}

El incremento de $I_{e}$ y $T_{\text {on }}$ tuvo una influencia pronunciada en el aumento de la rugosidad promedio de las superficies, tal como ha sido encontrado en varios trabajos de investigación [10,11]. Los resultados registrados en la Tabla 13 muestran un efecto reducido en el incremento de la rugosidad causado por la reducción de $\mathrm{T}_{\text {off }} \mathrm{y}$ del diámetro de electrodo.

\section{TABLA 13}

RUGOSIDAD: VALORES MEDIOS MÉDIA Ra $(\mu \mathrm{m})$

\begin{tabular}{lccccc} 
VARIABLE E & \multicolumn{4}{c}{ Nivel } & \multirow{\Delta}{*}{ Rank } \\
\cline { 2 - 4 } & $\mathbf{1}$ & $\mathbf{2}$ & $\mathbf{3}$ & & \\
\hline Diámetro & 3,869 & 3,688 & 3,608 & 0,261 & 5 \\
$\mathrm{I}_{\mathrm{e}}$ & 3,11 & 3,867 & 4,189 & 1,079 & 1 \\
$\mathrm{~T}_{\text {on }}$ & 3,156 & 3,847 & 4,163 & 1,007 & 2 \\
$\mathrm{~T}_{\text {off }}$ & 3,898 & 3,653 & 3,615 & 0,283 & 4 \\
$\mathrm{D}_{\text {af }}$ & 3,598 & 4,072 & 3,496 & 0,576 & 3 \\
$\mathrm{~T}_{\text {er }}$ & 3,688 & 3,693 & 3,784 & 0,096 & 7 \\
$\mathrm{~V}_{\text {gap }}$ & 3,791 & 3,655 & 3,72 & 0,136 & 6 \\
\hline
\end{tabular}

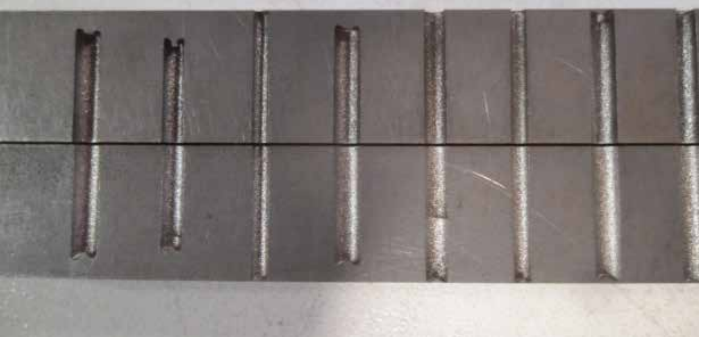

Fig. 6. Rugosidad en agujeros $2.5,2$ y $1.5 \mathrm{~mm}$. 
La mínima rugosidad superficial en el experimento $(2,82 \mu \mathrm{m})$ fue obtenida bajo las condiciones del test 22 . Mediante el análisis de $S / N$ fue predicho que podría realizarse una perforación con mínima rugosidad superficial, utilizando la combinación de variables $\mathrm{A}_{3} \mathrm{~B}_{1} \mathrm{C}_{1} \mathrm{D}_{3} \mathrm{E}_{3} \mathrm{~F}_{2} \mathrm{G}_{2}$. El resultado obtenido y el grado de optimización están registrados en la Tabla 9.

\section{E. Profundidad de perforación}

El ajuste de las variables de mecanizados utilizadas en el experimento permitió que la mayoría de las perforaciones realizadas $(59,26 \%)$ fueran pasantes. El análisis de los valores de $S / N$ indicó que las variables con mayor efecto sobre la profundidad de las perforaciones fueron Ie, diámetro del electrodo y $\mathrm{T}_{\text {on }}$. El grado de relevancia de cada variable $(\Delta)$ y el valor de $S / N$ para cada uno de sus niveles están registrados en la Tabla 14 y en la Fig. 7.

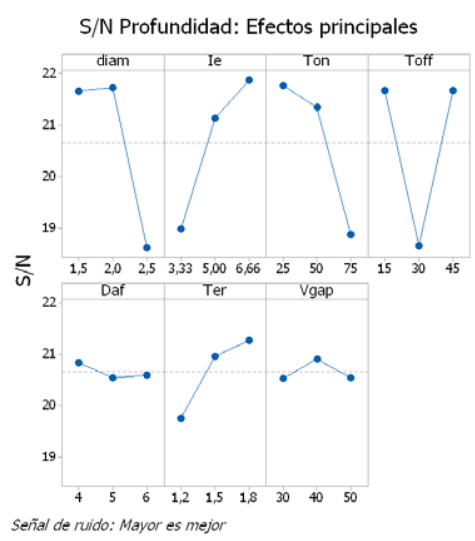

Fig. 7. S/N Profundidad.

Los electrodos de menor diámetro en combinación con bajos valores de $\mathrm{T}_{\text {on }}$ produjeron perforaciones con mayor profundidad promedio. La utilización del menor nivel de $\mathrm{I}_{\mathrm{e}}$, en combinación con el mayor nivel de $\mathrm{T}_{\text {on }}$, produjo mecanizado inestable y, como consecuencia, las perforaciones con menor profundidad. $\mathrm{D}_{\mathrm{af}} \mathrm{y} \mathrm{V}_{\text {gap }}$ tuvieron un efecto imperceptible sobre la profundidad de las perforaciones.
TABLA 14

S/N PROFUNDIDAD

S/N PROFUNDIDAD

\begin{tabular}{|c|c|c|c|c|c|}
\hline \multirow{2}{*}{ Variable } & \multicolumn{3}{|c|}{ Nivel } & & \multirow{2}{*}{ Rank } \\
\hline & 1 & 2 & 3 & & \\
\hline Diâmetro & 21,648 & 21,717 & 18,626 & 3,091 & 1 \\
\hline $\mathrm{T}_{\text {off }}$ & 21,664 & 18,657 & 21,67 & 3,013 & 2 \\
\hline $\mathrm{T}_{\text {on }}$ & 21,765 & 21,347 & 18,878 & 2,887 & 3 \\
\hline $\mathrm{I}_{\mathrm{e}}$ & 18,99 & 21,13 & 21,87 & 2,88 & 4 \\
\hline $\mathrm{T}_{\mathrm{er}}$ & 19,76 & 20,96 & 21,271 & 1,511 & 5 \\
\hline $\mathrm{V}_{\text {gap }}$ & 20,532 & 20,912 & 20,547 & 0,38 & 6 \\
\hline D & 20,843 & 20,547 & 20,6 & 0,296 & 7 \\
\hline
\end{tabular}

IV. Conclusiones

El desarrollo del experimento permitió concluir que la utilización de la metodología Taguchi es adecuada para la realización de experimentos en los cuales se requiere estudiar el efecto de múltiples variables sobre la respuesta de un sistema. Los resultados obtenidos permiten concluir lo siguiente:

- La MRR se incrementa con el aumento de $\mathrm{I}$, de diámetro del electrodo, con la reducción de $\mathrm{T}_{\text {off }} \mathrm{y}$ con la utilización de un valor de $\mathrm{T}_{\text {er }}$ ajustado.

- El efecto de las variables de mecanizado sobre la velocidad de avance de perforación es similar al efecto sobre la $M R R$, con la diferencia de que la reducción del diámetro de electrodo posibilita mayor velocidad de avance.

- Debido a que el proceso de perforación de agujeros profundos de pequeño diámetro requiere de la utilización de tiempos cortos de $\mathrm{T}_{\text {on }}$, el desgaste promedio sufrido por los electrodos fue considerablemente alto.

- $\quad$ El mínimo valor de TWR fue obtenido utilizando bajo nivel de Ie, elevado valor de $\mathrm{T}_{\text {on }} \mathrm{y}$ el mayor Nivel de diámetro de electrodo. Durante el experimento fue observado que la combinación de bajo nivel de $\mathrm{I}_{\mathrm{e}} \mathrm{y}$ duración prolongada de $\mathrm{T}_{\text {on }}$ causa inestabilidad en el mecanizado, impidiendo terminar las perforaciones. El ajuste para minimizar el desgaste del electrodo no permite realizar perforaciones profundas. 
Una perforación de máxima profundidad es influenciada por la utilización de un nivel elevado de $I_{e}$ en combinación con una baja duración de $T_{\text {on }}$.

\section{REFERENCIAS}

[1] P.C. Pande and H. S. Shan, Modern Machining Processes. New Delhi: Tata McGraw Hill Publications Ltd, 2008.

[2] N. Mohd, D. G. Solomon and Md. Fuad Bahari, "A review on current research trends in electrical discharge machining (EDM)", International Journal of Machine Tools and Manufacture, vol. 47 (7-8), pp. 1214-1228, Jun. 2007. DOI: http:// dx.doi.org/10.1016/j.ijmachtools.2006.08.026.

[3] M.Cao, Y.Hao, Y.Cao and S. Yang, "Mechanism and Experimental Research on Small-Hole EDM with $\mathrm{Cu}-\mathrm{Cr}$ Composite Electrode", Sensors \& Transducers, vol. 174 (7), pp. 268272, Jul. 2014.

[4] P. J. Ross, "Projeto por Parâmetros por Tolêrancias", Aplicações das tecnicas Taguchi na Engenharia da Qualidade. São Paulo, Brasil: Mc-Graw Hill, 1991.

[5] M. Simon and L. Grama, "Studies for obtaining a Small Hole, Rapid EDM Drilling Machine", Scientific Bulletin of the Petru Maior University of Targu Mures, vol. 8 (2), pp. 189-191, Dec. 2011.

[6] M. M. Rahman, M. A. R. Khan, K. Kadirgama, M. M. Noor and R. A. Bakar, "Experimental Investigation into Electrical Discharge
Machining of Stainless Steel 304", Journal of Applied Sciences, vol. 11 (3), pp. 549554, 2011. DOI: http://dx.doi.org/10.3923/ jas.2011.549.554.

[7] S. Hayakawa, Y. Sasaki, F. Itoigawa and T. Nakamura, "Relationship between occurrence of material removal and bubble expansion in electrical discharge machining", Procedia CIRP 6, pp. 174-179, 2013. DOI: http://dx.doi. org/10.1016/j.procir.2013.03.095.

[8] H. Takezawa, Y. Ito and N. Mohri, "The Behavior of Thin Electrode Wear in Electrical Discharge Machining", 13th International Symposium for Electromachining ISEM XIII, pp. 727-735, Bilbao, España, 2001.

[9] M. Ndaliman, A. Khan and M. Ali, "Influence of dielectric fluids on surface properties of electrical discharge machined titanium alloy", Proceedings of the Institution of Mechanical Engineers Part B Journal of Engineering Manufacture, vol. 227 (9), pp. 1310-1316, Sep. 2013. DOI: http://dx.doi. org/10.1177/0954405413488592.

[10] C. Purcar and C. Simion, "Studies about the roughness of the surfaces machined by EDM", Nonconventional Technologies Review, pp. 7881, Jun. 2012.

[11] S. S. Habib, "Study of the parameters in electrical discharge machining through response surface methodology approach", Applied Mathematical Modelling, vol. 33 (12), pp. 4397-4407, Dec. 2009. DOI: http://dx.doi. org/10.1016/j.apm.2009.03.021. 